\title{
Amoebic Liver abscess in a child communicating with colon: A rare complication
}

\author{
Anmol Bhatia ${ }^{1 *}$, Kushaljit Singh Sodhi², Babu Ram Thapa ${ }^{1}$ \\ ${ }^{1}$ Department of Gastroenterology, Institute of Medical Education and Research, Sector-12, Chandigarh, India. \\ ${ }^{2}$ Radiodiagnosis and Imaging, Post Graduate Institute of Medical Education and Research, Sector-12, Chandigarh, India.
}

Received: October 24, 2016; Accepted: December 12, 2016; Published: January 28, 2017

*Corresponding author: Anmol Bhatia, Assistant Professor, GE Radiology Section, Department of Gastroenterology, Post Graduate Institute of Medical Education and Research, Chandigarh, India; Tel: 0091-9914201986; E-mail: anmol_bhatia26@yahoo.co.in

A 3-year old boy presented with complaints of fever and abdominal pain for three days. Ultrasound of abdomen showed a liver abscess measuring $3.5 \times 2.8 \mathrm{cms}$ in segment 5 with a possible communication with gut loop. Contrast enhanced computed tomography of the abdomen showed a peripherally enhancing abscess with air foci and a fistulous communication with ascending colon (Figure 1). A surgical opinion was taken and ultrasound guided diagnostic aspiration was done, which yielded around $10 \mathrm{ml}$ of pus. The pus was sent for microbiological examination and amoebic serology was positive. The child was started on intravenous antibiotics. Follow up ultrasound done

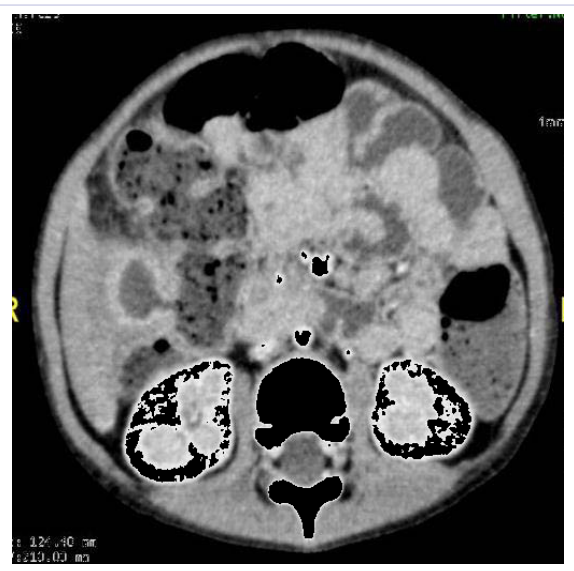

Figure 1a: Axial CT images showing peripherally enhancing hypodense lesion in the right lobe of the liver with a breach along its medial aspect and communication with the colon. one week later showed reduced size of abscess, with persistent communication with bowel. The child was later discharged in a febrile state and started on oral antibiotics. Ultrasound done two months later showed only mild heterogeneity of liver parenchyma where abscess was initially present, but no residual lesion was seen. The child is asymptomatic and on a regular follow up.

Declaration: Informed consent was obtained from the patient for the publication of imaging

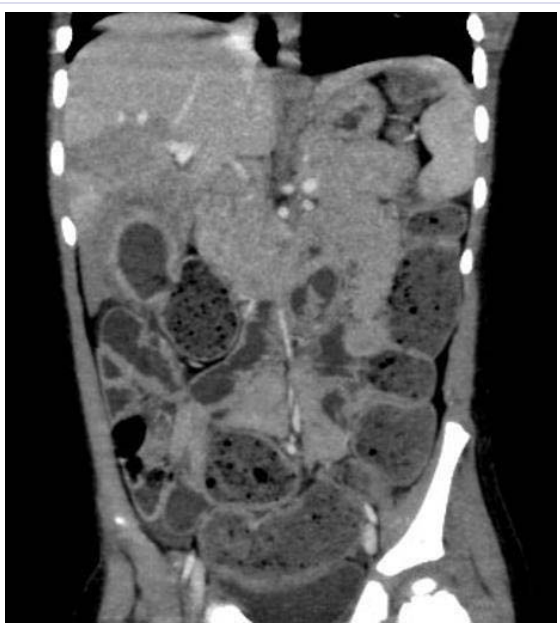

Figure 1b: Coronal (B) CT images showing peripherally enhancing hypodense lesion in the right lobe of the liver with a breach along its medial aspect and communication with the colon.

Citation: Anmol Bhatia, et.al.(2017) Amoebic Liver abscess in a child communicating with colon: A rare complication. Gastroenterol Pancreatol Liver Disord 4(2):1-1. DOI: http://dx.doi.org/10.15226/2374-815X/4/2/00184. 